\title{
Improvement of and Parameter Identification for the Bimodal Time-Varying Modified Kanai-Tajimi Power Spectral Model
}

\author{
Huiguo Chen, Ting Zhong, Guocui Liu, and Junru Ren \\ Department of Civil Engineering, Logistical Engineering University, Chongqing 401311, China \\ Correspondence should be addressed to Ting Zhong; zhongting2015@126.com
}

Received 15 January 2017; Revised 7 June 2017; Accepted 11 July 2017; Published 10 August 2017

Academic Editor: M. I. Herreros

Copyright (C) 2017 Huiguo Chen et al. This is an open access article distributed under the Creative Commons Attribution License, which permits unrestricted use, distribution, and reproduction in any medium, provided the original work is properly cited.

\begin{abstract}
Based on the Kanai-Tajimi power spectrum filtering method proposed by Du Xiuli et al., a genetic algorithm and a quadratic optimization identification technique are employed to improve the bimodal time-varying modified Kanai-Tajimi power spectral model and the parameter identification method proposed by Vlachos et al. Additionally, a method for modeling time-varying power spectrum parameters for ground motion is proposed. The 8244 Orion and Chi-Chi earthquake accelerograms are selected as examples for time-varying power spectral model parameter identification and ground motion simulations to verify the feasibility and effectiveness of the improved bimodal time-varying modified Kanai-Tajimi power spectral model. The results of this study provide important references for designing ground motion inputs for seismic analyses of major engineering structures.
\end{abstract}

\section{Introduction}

Severe ground motion is an extremely complex process. Affected by factors such as the seismic source mechanism, the travel path, and the field characteristics, observed ground motions demonstrate prominent nonstationary characteristics in both the time and the frequency domain. Among the known seismic characteristics that have significant impacts on the seismic response of a structure, in addition to its nonstationary strength characteristics, the impact of nonstationary seismic frequency characteristics on a structure's nonlinear seismic response has become a focus in recent years. Research by Yeh and Wen [1] showed that nonstationary seismic frequencies have substantial impacts on rigid structures as their strength degrades; Cao et al. [2]. believed that concentrated seismic energy in the time domain had a considerably unfavorable effect on a structure. Research by Conte and Wang suggested that, due to the structural damage caused by ground motion, the structural period is prolonged; when the long-period seismic component after a strong earthquake segment is close to a structure's prolonged period, "instantaneous resonance" can occur, and the damage to the structure becomes more severe or even exceeds that due to a strong earthquake $[3,4]$. Therefore, creating a method for modeling nonstationary ground motion in which the characteristics of the simulated ground motion are similar to those of the characteristic time-frequency distribution of actual ground motion has become a realistic and important topic in seismic research.

In 1965, Priestley proposed evolutionary stochastic process [5] theory in which a nonstationary process is converted to an integral of a stationary process and a deterministic modulation function, and then, the time-varying spectrum density of the nonstationary process is obtained from the spectrum density of the stationary process, and a method of describing the characteristics of the spectrum of a nonstationary process and a model of nonstationary ground motion is created. However, in this method, to simulate ground motion with a fully nonstationary frequency, a time-varying power spectral model is required. For many years, researchers worldwide conducted various studies of time-varying power spectral models for ground motion. For instance, in 1975, Kameda [6] employed a multifilter technique, recorded the power spectrum of an actual earthquake that varied over time and with frequency, and created a time-varying power spectral model; Sugito et al. [7] performed a statistical analysis of 118 actual earthquakes in Japan using the Kameda model and provided a typical time-varying power spectrum attenuation 
relationship. In 1988, Deodatis [8] analyzed the Niigata earthquake's field liquefaction characteristics in Japan, modified the time-varying property of the Kanai-Tajimi model, and simulated ground motion with a fully nonstationary timefrequency based on the characteristics of time-varying power spectrum of the ground motion associated with the Niigata earthquake. As modern time-frequency signal analysis develops, the methods of describing time-varying power spectra continue to evolve. In 2010, Fan estimated the time-varying spectrum of an actual strong earthquake using harmonic wavelet transformation and performed a statistical analysis of the characteristics of the time-varying spectra of seismic waves at various sites [9]; he also created a modified KanaiTajimi nonstationary stochastic model with time-varying seismic waves by means of nonlinear fitting. In 2015, based on a power spectrum density model for stationary seismic processes, Liu et al. [10] developed a general evolutionary spectral model for fully nonstationary time-frequency seismic processes based on the Specification for Seismic Design of Hydraulic Construction in China; values for the parameters of this model were determined. In 2016, based on the theory that there is a time gap between when the P, $S$, and surface waves in a seismic wave reach the recording field and when the earthquake releases energy via component waves of various frequencies, Vlachos et al. [11] created a time-varying power spectral model for ground motion using a bimodal time-varying modified Kanai-Tajimi model. This model accurately described characteristics of fully nonstationary ground motion and was an attempt to model the time-varying power spectrum of actual ground motion. In this paper, based on work by Vlachos, a genetic algorithm is employed to perform identify optimal parameters for the bimodal time-varying modified Kanai-Tajimi power spectral model proposed by Vlachos. In combination with the filtering technique proposed by Xiu-Li and Hou-Qun [12], a method is created to describe and model the nonstationary timefrequency characteristics of actual ground motion. Additionally, the method of simulating ground motion with a nonstationary time-frequency proposed by Z.-J. Liu and L. Liu [13] is employed to verify the effectiveness of the bimodal time-varying modified Kanai-Tajimi power spectral model and parameter identification method.

\section{Bimodal Time-Varying Modified Kanai-Tajimi Power Spectral Model and Improvement}

2.1. Fundamental Theory of the Evolutionary Spectrum Method. According to evolutionary spectrum density-based model of nonstationary stochastic processes proposed by Priestley [5], any nonstationary stochastic process $y(t)$ with an average value of zero is defined as

$$
y(t)=\int_{-\infty}^{+\infty}(\omega, t) e^{i \omega t} d F_{x}(\omega),
$$

where $F_{x}(\omega)$ is a complex stationary stochastic process with an orthogonal increment and $A(\omega, t)$ is a slowly varying modulation function.
Assume that a stationary process $x(t)$ satisfies

$$
x(t)=\int_{-\infty}^{\infty} e^{i \omega t} d F_{x}(\omega) .
$$

Then, the evolutionary spectrum function (the timevarying or progressive power spectrum) of $y(t)$ is represented as

$$
S(\omega, t)=|A(\omega, t)|^{2} S_{x}(\omega) d \omega,
$$

where $S_{x}(\omega)$ is the power spectrum of stationary process $x(t)$.

Based on the above evolutionary spectrum theory, a numerical simulation of a nonstationary stochastic process, $y(t)$, was initially proposed by Shinozuka et al. $[14,15]$ and was improved continuously until the following final ground motion simulation formula was reached:

$$
y(t)=2 \sum_{k=1}^{n} \sqrt{S(\omega, t) \Delta \omega \cos \left(\omega_{k} t+\varphi_{k}\right)},
$$

where $\varphi_{k}$ is a random phase that is evenly distributed on $[0,2 \pi]$.

Equations (3) and (4) show that the power spectrum density function, $S(\omega, t)$, is an important index for the characteristics of the time-frequency of ground motion.

\subsection{Bimodal Time-Varying Modified Kanai-Tajimi Spectral} Model. In a stochastic model of early stage ground motion, due to the difficulty of creating a fully time-varying power spectral model, researchers prefer to generate a stochastic process from the power spectrum of a stationary stochastic process and then to modify the nonstationary characteristics of this stochastic process to simulate ground motion. Based on this idea, Japanese researchers Kanai (1957) and Tajimi $(1960)[16,17]$ selected a soil layer on bedrock as a singledegree-of-freedom linear filter to investigate the effect of various types of soil layers on ground motion and proposed a well-known ground motion power spectral model, the KanaiTajimi model, shown as follows:

$$
S(\omega)=S_{0} \frac{1+4 \xi_{\mathrm{g}}^{2}\left(\omega / \omega_{g}\right)^{2}}{\left(1-\left(\omega / \omega_{g}\right)^{2}\right)^{2}+4 \xi_{\mathrm{g}}^{2}\left(\omega / \omega_{g}\right)^{2}},
$$

where $S_{0}$ is the power spectrum based on white noise excitation of the bedrock, $\omega_{g}$ is the characteristic frequency of the soil layer, and $\xi_{g}$ is the damping ratio of the single-degreeof-freedom linear filter.

This model suggests that an earthquake causes movement in the bedrock, which is then transmitted to the structure via the surface soil layer. The bedrock's acceleration is treated as white noise whose average is zero and whose spectrum density is $S_{0}$. The surface soil layer is treated as a filter. The Kanai-Tajimi power spectral model considers the effect of the surface soil layer's characteristics on the characteristics of the seismic frequency spectrum and is physically interpretable. Therefore, this model is widely adopted in seismic engineering. Because the Kanai-Tajimi power spectral model is 
not time-varying in the frequency domain, the Kanai-Tajimi power spectrum is unable to directly model ground motion with fully nonstationary time-frequency characteristics. To make the conventional Kanai-Tajimi power spectrum capable of representing a time-varying seismic power spectrum, Dedatis et al. [8] proposed a time-varying modification of the Kanai-Tajimi power spectral model. Vlachos et al. [11] optimized the resulting time-varying modified Kanai-Tajimi power spectral model, superposed the conventional singlepeak Kanai-Tajimi equation, and proposed the following bimodal fully nonstationary time-varying Kanai-Tajimi spectral model:

$$
\begin{aligned}
& S(\omega, t)=|H(\omega)|^{2} \sum_{k=1}^{k=2} S_{0}^{(k)}(t) \\
& \cdot \frac{1+\left(2 \xi_{g}^{(k)}(t) \omega / \omega_{g}^{(k)}(t)\right)^{2}}{\left(1-\left(\omega / \omega_{g}^{(k)}(t)\right)^{2}\right)^{2}+\left(2 \xi_{g}^{(k)}(t) \omega / \omega_{g}^{(k)}(t)\right)^{2}},
\end{aligned}
$$

where $S(\omega, t)$ is a time-varying power spectrum for frequency and time, $H(\omega)$ is a filter, and $\omega_{g}^{(k)}(t), \xi_{g}^{(k)}(t)$, and $S_{0}^{(k)}(t)$ are the characteristic frequency, damping ratio, and modal participant factor, respectively, under the $k$ th modal condition in the time-varying model.

2.3. Improvement of the Bimodal Time-Varying Modified Kanai-Tajimi Spectral Model. In (6), the selection of the filter, $H(\omega)$, is critical. The reason is that there are two essential limitations to the application of the Kanai-Tajimi power spectral model. The first one is on the process energy. Research by Jin-Ren et al. [18] showed that, to satisfy the requirement of limited seismic process energy, the process variance of the ground motion acceleration and the variances of the first- and second-order derivative processes should be limited. However, based on the rules for the convergence of general integrals, the variance of the acceleration process in the Kanai-Tajimi power spectral model is unbounded. To satisfy the condition of the process energy being limited, the Kanai-Tajimi power spectral model should be filtered and modified. The second limitation is that the energy must be zero when the frequency is zero. In the Kanai-Tajimi power spectral model, when $\omega$ approaches zero, the ground velocity and displacement become infinite, which is obviously unrealistic. Therefore, to solve the problem of nonzero energy when the frequency is zero frequency in the conventional Kanai-Tajimi power spectral model and to address longperiod noise pollution in the modified simulation signal, the Kanai-Tajimi power spectral model should be filtered and modified. However, improvements to the Kanai-Tajimi spectrum in the past only addressed a single aspect, which focused on the acceleration's boundary integral or derivative process; few considered both. When creating a time-varying Kanai-Tajimi spectral model, Vlachos et al. [11] employed a fourth-order Butterworth high-pass filter. Because of the Butterworth high-pass filter's characteristics, its higher-order characteristics may result in pseudo-filtering behavior and prolong harmful low-amplitude zero-average oscillations in the signal's pulse response. Therefore, adding a high-pass filter improves the boundary integral process but does not improve the derivative process. However, adding a low-pass filter improves the boundary derivative process but has no effect on the integral process. Additionally, when only the order of the low-pass filter is increased, the power spectrum of the integral process still has a singular point [18]. To resolve this dilemma, Xiu-Li and Hou-Qun [12] investigated the simplified seismic acceleration Fourier spectrum proposed by Ming-Zheng, which considers the propagation path and the effect of local field conditions [19], and proposed a method for filtering the Kanai-Tajimi spectrum using concatenated lowpass and high-pass filters. This method effectively combines the characteristics of low-pass and high-pass filters, satisfies the high- and low-frequency requirements of the power spectrum, and is an ideal method for improving the Kanai-Tajimi spectral model of ground motion. To guarantee accuracy in the Kanai-Tajimi power spectrum identification process, this paper improves the bimodal time-varying modified KanaiTajimi model created by Vlachos et al. [11] by replacing the filter in the model with the filter model proposed by Xiu-Li and Hou-Qun [12]. The filter is as follows:

$$
|H(\omega)|^{2}=\frac{1}{1+(D \omega)} \frac{\omega^{2}}{\omega^{2}+\omega_{h}^{2}},
$$

where $D$ is a spectrum parameter that reflects the bedrock's characteristics and $\omega_{h}$ is the low corner frequency.

$D$ and $\omega_{h}$ have clear physical interpretations. Mijatovic (2013) [20] proposed that $D$ be 0.03 second and $\omega_{h}$ be as follows:

$$
\omega_{h}=\frac{2 \pi \beta}{r},
$$

where $r$ is the radius of an equivalent disc and $\beta=3.5 \mathrm{~km} / \mathrm{s}$. Ming-Zheng (1986) [19] provided a statistical relationship between $r$ and the earthquake's magnitude. A simple way to determine $\omega_{h}$ is

$$
\omega_{h}=\frac{2 \pi}{T_{r}}
$$

where $T_{r}$ is the duration of the fault crack. Ying (1987) [21] identified a statistical relationship between $T_{r}$ and an earthquake's magnitude, $M$ :

$$
\log _{10} T_{r}=d_{1}+d_{2} \cdot M
$$

where $d_{1}=-1.325, d_{2}=0.353$. Here, $T_{r}$ is equivalent to $90 \%$ of the duration of energy at the epicenter.

The improved bimodal time-varying modified KanaiTajimi model is as follows:

$$
\begin{aligned}
& S(\omega, t)=\frac{1}{1+(D \omega)} \frac{\omega^{2}}{\omega^{2}+\omega_{h}^{2}} \sum_{k=1}^{k=2} S_{0}^{(k)}(t) \\
& \cdot \frac{1+\left(2 \xi_{g}^{(k)}(t) \omega / \omega_{g}^{(k)}(t)\right)^{2}}{\left(1-\left(\omega / \omega_{g}^{(k)}(t)\right)^{2}\right)^{2}+\left(2 \xi_{g}^{(k)}(t) \omega / \omega_{g}^{(k)}(t)\right)^{2}}
\end{aligned}
$$




\section{Model Parameter Identification}

3.1. Introduction of the Genetic Algorithm. When creating a nonstationary time-frequency model of ground motion, the key is to identify the parameters of the resulting time-varying power spectral model via numerical analysis to obtain a timevarying seismic power spectrum. The bimodal time-varying modified Kanai-Tajimi model has six unknown parameters, $\omega_{g}^{(1)}(t), \xi_{g}^{(1)}(t), \omega_{g}^{(2)}(t), \xi_{g}^{(2)}(t), S_{0}^{(1)}(t), S_{0}^{(2)}(t)$. Identifying these six parameters is a typical multiparameter and nonlinear optimization problem. Among current parameter identification methods and numerical optimization methods, the genetic algorithm is based on the theory of biological evolution; it simulates a biological evolutionary process by producing a next-generation solution via operations such as replication, intersection, and mutation and gradually eliminates solutions with low fitness values to obtain a solution with a high fitness value. This method was initially proposed in 1975 by Professor Holland of the University of Michigan in the United States [22]. Since then, it has been widely adopted in various fields, such as optimization identification, machine learning, artificial intelligence control, image processing, and pattern recognition. The basic idea underlying its application in optimization identification is to treat a solution to an actual problem as a representation of a biological individual and to treat the process of solving an optimization problem as the genetic process of a species obtaining an optimal biological individual, where the representation of the optimal biological individual is the optimal solution. Because this is essentially a stochastic probability search algorithm and has the characteristics of an adaptive global search, it is ideal for solving multiparameter and nonlinear planning problems. Therefore, this paper introduces this method as the primary means of identifying the parameters of the improved bimodal modified Kanai-Tajimi model.

3.2. Model Parameter Identification and Fitting. Research by Vlachos et al. [11] suggested that, in the bimodal modified Kanai-Tajimi model, the spectrum strength factors, $S_{0}^{(1)}(t)$ and $S_{0}^{(2)}(t)$, have no direct relationship with the spectral energy of each mode; their values only represent linear combinations of the modes. Therefore, the following ratio is defined to replace the absolute values:

$$
S_{0}^{(k)}(t)=\frac{S_{0}^{(k)}(t)}{S_{0}^{(1)}(t)}
$$

According to (12), when $S_{0}^{(1)}(t)$ is fixed, that is, when $S_{0}^{(1)}(t)=$ 1 , the number of parameters to be identified in the model is reduced to five; that is, $X_{t}=\left\{\omega_{g}^{(1)}(t), \xi_{g}^{(1)}(t), \omega_{g}^{(2)}(t)\right.$, $\left.\xi_{g}^{(2)}(t), S_{0}^{(2)}(t)\right\}$. To satisfy the requirements of the global optimization method in a genetic algorithm, the least squaresbased model parameter identification fitness function is as follows:

$$
X_{t}=\underset{x_{t}}{\operatorname{argmin}} \int_{-\infty}^{+\infty}\left(S_{1}(\omega, t)-S\left(\omega, t, x_{t}\right)\right)^{2} d \omega,
$$

where $S_{1}(\omega, t)$ is the time-varying power spectrum of the actual ground motion, which is obtained via signal timefrequency analysis, and $S\left(\omega, t, x_{t}\right)$ is the time-varying power spectral model of the ground motion represented by (11).

MATLAB's genetic algorithm toolbox (Mijatovic [20]) is employed to identify $X_{t}$ and to obtain the values of parameters $\left(X_{t i}\right)=\left\{\omega_{g}^{(1)}\left(t_{i}\right), \xi_{g}^{(1)}\left(t_{i}\right), \omega_{g}^{(2)}\left(t_{i}\right), \xi_{g}^{(2)}\left(t_{i}\right), S_{0}^{(2)}\left(t_{i}\right)\right\}$ in an instantaneous frequency curve model at each time point $t_{i}$ of the ground motion's time-varying power spectrum. Among the identified parameters, $\xi_{g}^{(1)}, \xi_{g}^{(2)}$ are defined as damping ratios of the top soil layer on the bedrock, which are normally constants. To simplify the analysis, the values of parameters $\xi_{g}^{(1)}, \xi_{g}^{(2)}$ are set to the mean value of $\left\{\xi_{g}^{(1)}\left(t_{i}\right), \xi_{g}^{(2)}\left(t_{i}\right)\right\}$ identified at each time $t_{i}$ :

$$
\begin{aligned}
& \zeta_{g}^{(1)}=\overline{\left\{\zeta_{g}^{(1)}(t), t \in\left[0, T_{d}\right]\right\}}, \\
& \zeta_{g}^{(2)}=\overline{\left\{\zeta_{g}^{(2)}(t), t \in\left[0, T_{d}\right]\right\}} .
\end{aligned}
$$

Next the five original unknown parameters, $X_{t}=\left\{\omega_{g}^{(1)}(t)\right.$, $\left.\xi_{g}^{(1)}(t), \omega_{g}^{(2)}(t), \xi_{g}^{(2)}(t), S_{0}^{(2)}(t)\right\}$, are reduced to three, which are $X_{t 1}=\left\{\omega_{g}^{(1)}(t), \omega_{g}^{(2)}(t), S_{0}^{(2)}(t)\right\} . S_{0}^{(1)}(t), \xi_{g}^{(1)}, \xi_{g}^{(2)}$, which are determined by (12) and (14), and are treated as known parameters, and a genetic algorithm is employed to perform quadratic optimization of $X_{t 1}$ to identify the values of the remaining parameters in the model at each time point $t_{i}$; they are recorded as $\left(X_{t 1 i}\right)=\left\{\omega_{g}^{(1)}\left(t_{i}\right), \omega_{g}^{(2)}\left(t_{i}\right), S_{0}^{(2)}\left(t_{i}\right)\right\}$. These identified parameters $\left(X_{t 1 i}\right)$ are a series of scattered points at time point $t_{i}$.

The resulting scattered point parameter values $\left(X_{t 1 i}\right)$ should be translated to mathematical expressions via modeling before they are used in ground motion simulations. Therefore, based on characteristics of the actual parameters identified for the ground motion time-varying power spectral model, this paper employs a Gaussian distribution function to fit the identified $\omega_{g}^{(1)}\left(t_{i}\right)$ and $\omega_{g}^{(2)}\left(t_{i}\right)$, whereas $S_{0}^{(2)}\left(t_{i}\right)$ is fitted using smooth splines. The resulting expressions for $\omega_{g}^{(1)}(t)$ and $\omega_{g}^{(2)}(t)$ are as follows:

$$
\omega_{g}^{(k)}(t)=F_{1}^{k} e^{\left(-\left(t-a_{1}^{k} / b_{1}^{k}\right)^{2}\right)}+F_{2}^{k} e^{\left(-\left(t-a_{2}^{k} / b_{2}^{k}\right)^{2}\right)} \quad(k=1,2),
$$

where $k=1,2$ and $F_{1}^{k}, a_{1}^{k}, b_{1}^{k}, F_{2}^{k}, a_{2}^{k}, b_{2}^{k}$ are the parameter values for the corresponding values of $k$.

Figure 1 is a diagram of the parameter identification process for the bimodal modified Kanai-Tajimi model. This diagram shows that, of the six model parameters, only $S_{0}^{(1)}(t)$ is defined directly; the other parameters are identified by the genetic algorithm. A quadratic optimization identification technique is employed during the identification process. First, a genetic algorithm is employed to identify each parameter, and the results are averaged to obtain values of the damping ratio parameters, $\xi_{g}^{(1)}, \xi_{g}^{(2)}$, for the cover soil layer. In quadratic optimization, values of $S_{0}^{(1)}(t), \xi_{g}^{(1)}, \xi_{g}^{(2)}$ are used to reidentify the values of the scattered points $\omega_{g}^{(1)}\left(t_{i}\right), \omega_{g}^{(2)}\left(t_{i}\right), S_{0}^{(2)}\left(t_{i}\right)$ at 


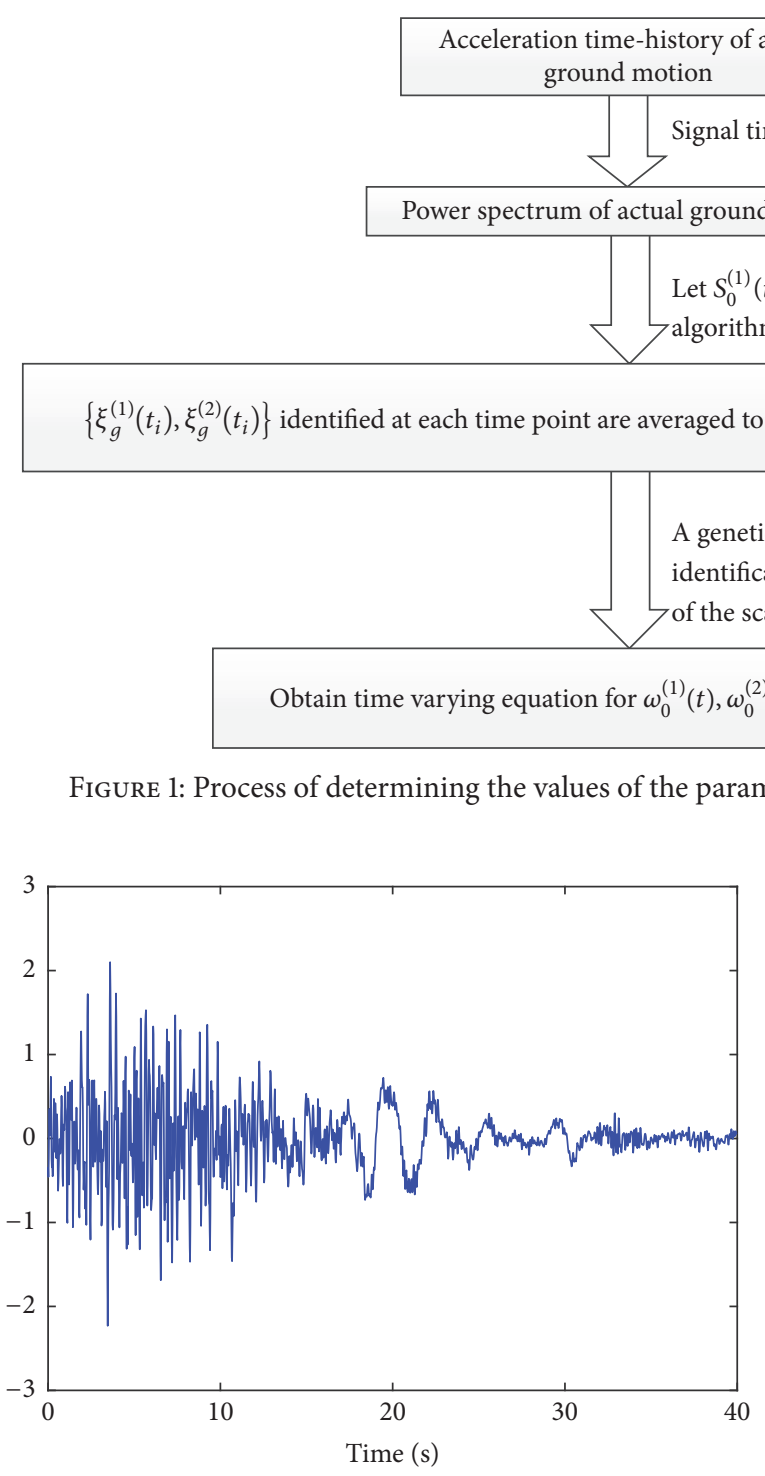

(a) 8244 Orion

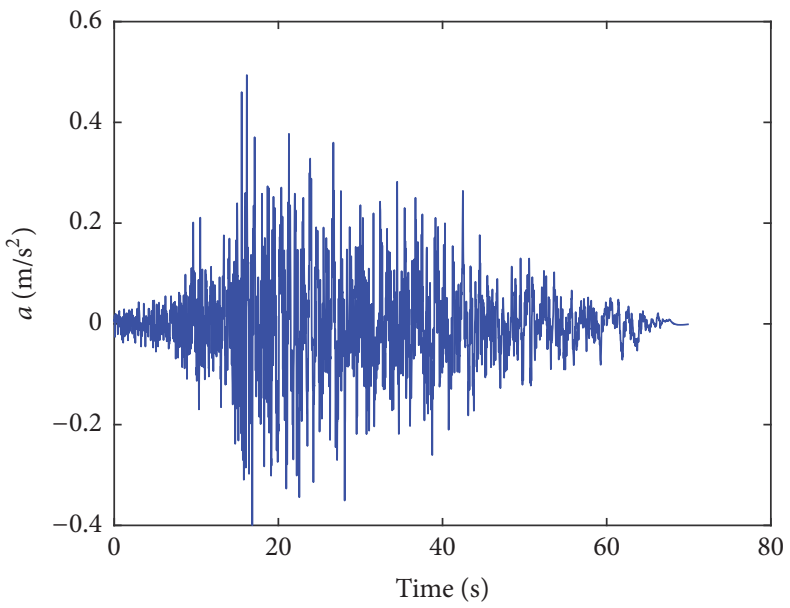

(b) Chi-Chi

FIGURE 2: Acceleration time-history diagrams for the 8244 Orion and Chi-Chi accelerograms.

each time point $t_{i}$. Then, based on the identified parameters, a time-varying equation relating $\omega_{g}^{(1)}(t), \omega_{g}^{(2)}(t), S_{0}^{(2)}(t)$ is obtained via fitting.

3.3. Example of Model Parameter Identification. The 8244 Orion (1971, San Fernando) and Chi-Chi 1999, station CHY102, NS component accelerograms seismic wave records are selected as examples for model parameter identification; their acceleration time-history curves are shown in Figure 2. Based on a study by Xiu-Li and Hou-Qun [12], the values of the filter parameters are as follows: $D$ is always 0.03 seconds and $\omega_{h}$ is 0.6214 for the 8244 Orion record and $\omega_{h}$ is 0.2844 for the Chi-Chi record. Short-time Fourier transformation and the Hanning window smoothing technique are combined to obtain time-varying power spectra for the acceleration time-histories of the two ground motions; they are shown in Figure 3.
Figure 4 shows the initial values of $\left\{\xi_{g}^{(1)}\left(t_{i}\right), \xi_{g}^{(2)}\left(t_{i}\right)\right\}$ from the genetic algorithm. The values for the 8244 Orion and Chi-Chi seismic waves, $\xi_{g}^{(1)}, \xi_{g}^{(2)}$, calculated using (14) are as follows:

8244 Orion

$$
\begin{aligned}
& \xi_{g}^{(1)}=0.23, \\
& \xi_{g}^{(2)}=0.42 .
\end{aligned}
$$

Chi-Chi

$$
\begin{gathered}
\xi_{g}^{(1)}=0.34, \\
\xi_{g}^{(2)}=0.26 .
\end{gathered}
$$




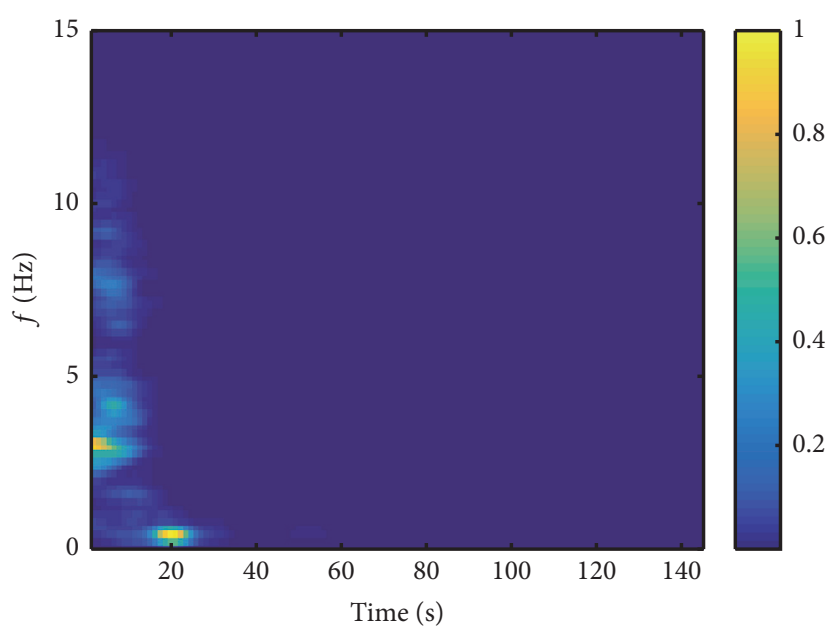

(a) 8244 Orion

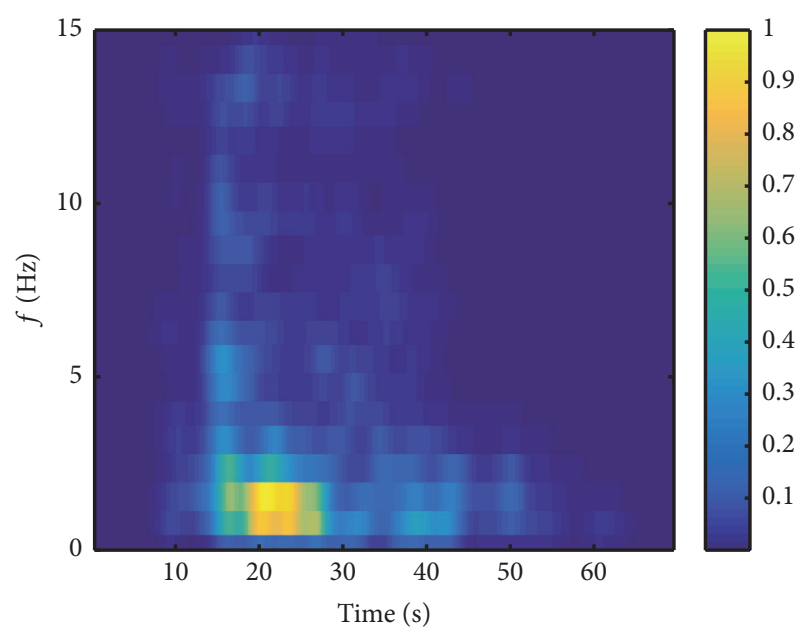

(b) Chi-Chi

FIgURE 3: Time-varying power spectra of the 8244 Orion and Chi-Chi accelerograms.

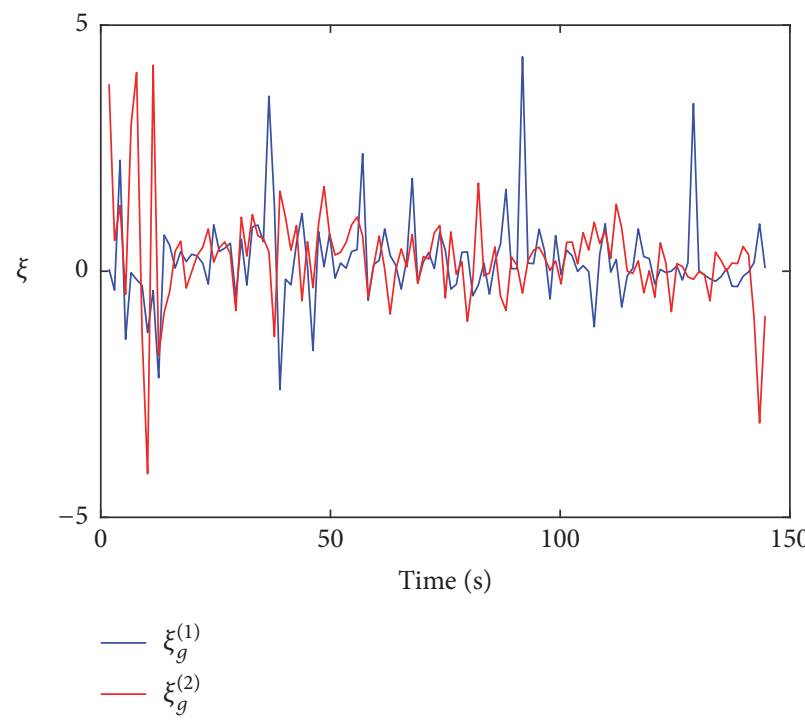

(a) 8244 Orion

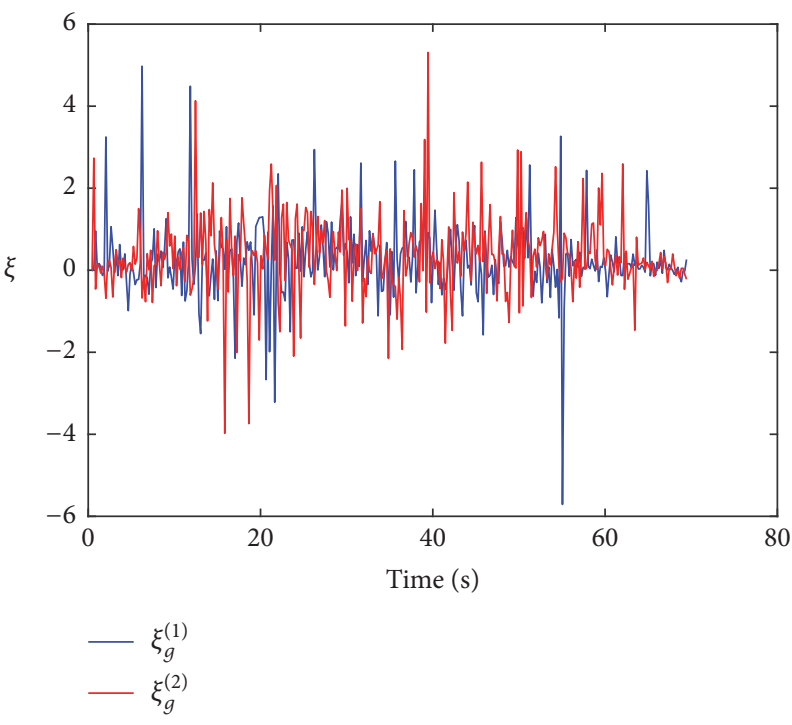

(b) Chi-Chi

FIGURE 4: Identified values of $\left\{\xi_{g}^{(1)}\left(t_{i}\right), \xi_{g}^{(2)}\left(t_{i}\right)\right\}$ for the 8244 Orion and Chi-Chi accelerograms.

Based on the mean of $\xi_{g}^{(1)}, \xi_{g}^{(2)}$ and genetic algorithmbased quadratic optimization, the identified values of the parameters $\omega_{g}^{(1)}\left(t_{i}\right), \omega_{g}^{(2)}\left(t_{i}\right), S_{0}^{(2)}\left(t_{i}\right)$ in the model at each time point $t_{i}$ are obtained; they are shown in Figures 5-7. The diagrams also include the curve fitted after parameter identification. Tables 1 and 2 list the final values identified for the model parameters $\omega_{g}^{(1)}\left(t_{i}\right), \omega_{g}^{(2)}\left(t_{i}\right), S_{0}^{(2)}\left(t_{i}\right)$ and their fitted time-varying expressions. The time-varying power spectra are obtained after modeling by inverse calculation based on the fitting parameters and the time-varying equation, as shown in Figure 8. Comparison of Figures 3 and 8 shows that the time-varying power spectral model effectively reflects the major nonstationary time-frequency characteristics of the original ground motion.

\section{Ground Motion Simulation Example Verification}

In Section 2.3, the parameters in bimodal time-varying modified Kanai-Tajimi model, $X_{t}=\left\{\omega_{g}^{(1)}(t), \xi_{g}^{(1)}(t), S_{0}^{(1)}(t), \omega_{g}^{(2)}(t)\right.$, $\left.\xi_{g}^{(2)}(t), S_{0}^{(2)}(t)\right\}$, are obtained via genetic algorithm-based optimization. However, the most direct and effective method of verifying the effectiveness of the bimodal time-varying modified Kanai-Tajimi model and the identified parameters in describing the nonstationary time-frequency characteristics of the ground motion is to perform an inverse calculation, analyze the ground motion based on the identified parameters, and compare the actual and simulated ground motions. Therefore, in this section, the effectiveness of model 


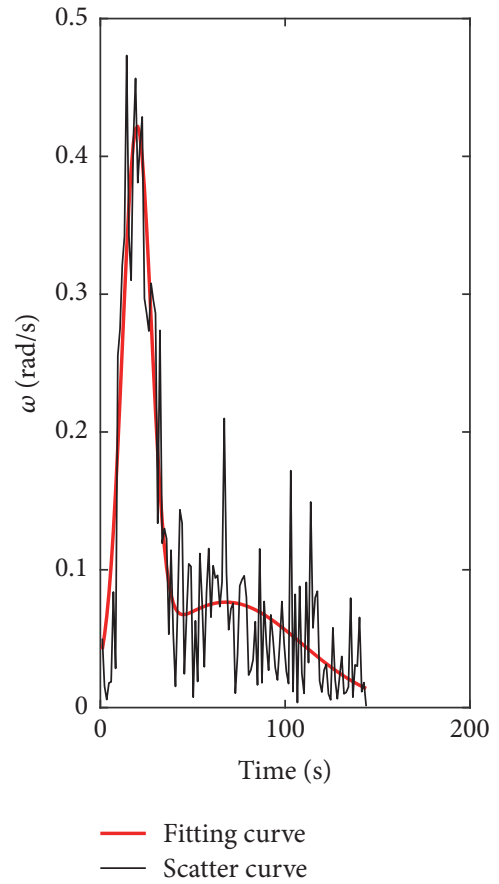

(a) $\omega_{g}^{(1)}(t)$

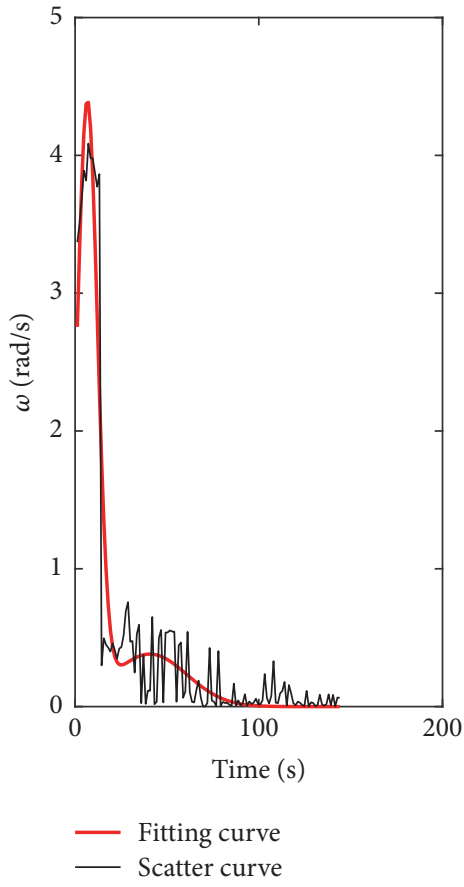

(b) $\omega_{g}^{(2)}(t)$

FigURE 5: Fitting of parameters $\omega_{g}^{(1)}(t), \omega_{g}^{(2)}(t)$ for the 8244 Orion accelerogram seismic wave.

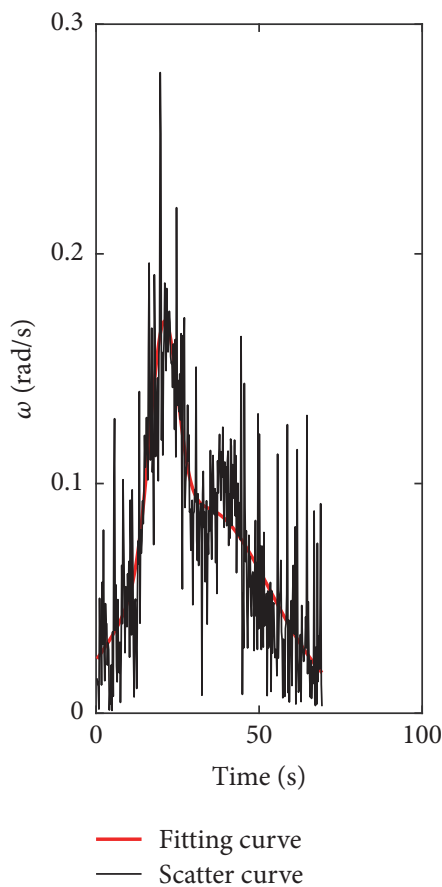

(a) $\omega_{g}^{(1)}(t)$

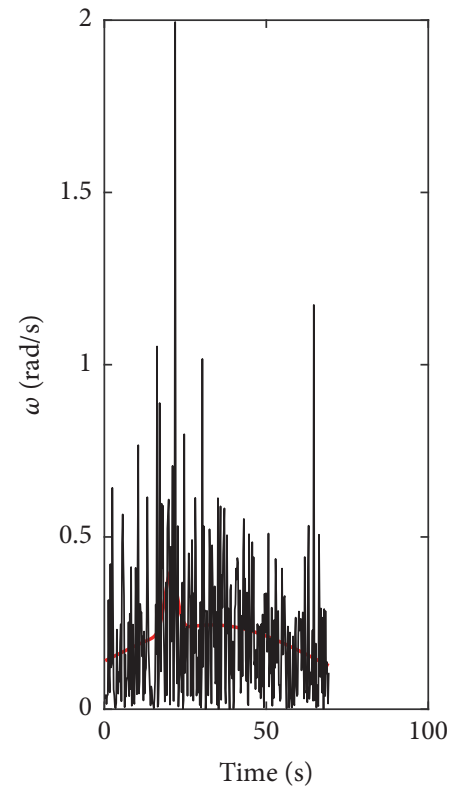

Fitting curve

Scatter curve

(b) $\omega_{g}^{(2)}(t)$

FiguRE 6: Fitting of parameters $\omega_{g}^{(1)}(t), \omega_{g}^{(2)}(t)$ for the Chi-Chi accelerogram seismic wave. 


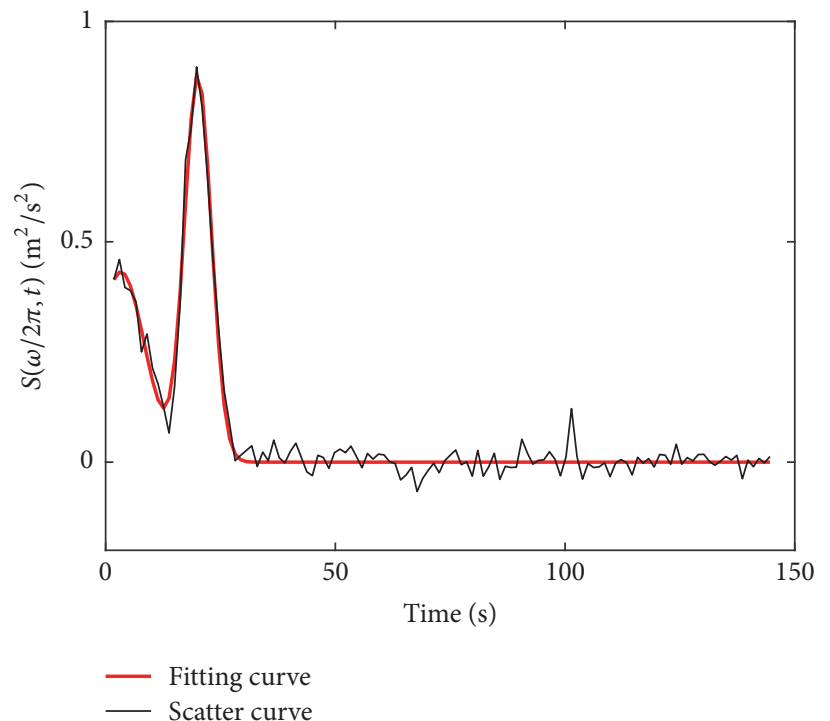

(a) 8244 Orion

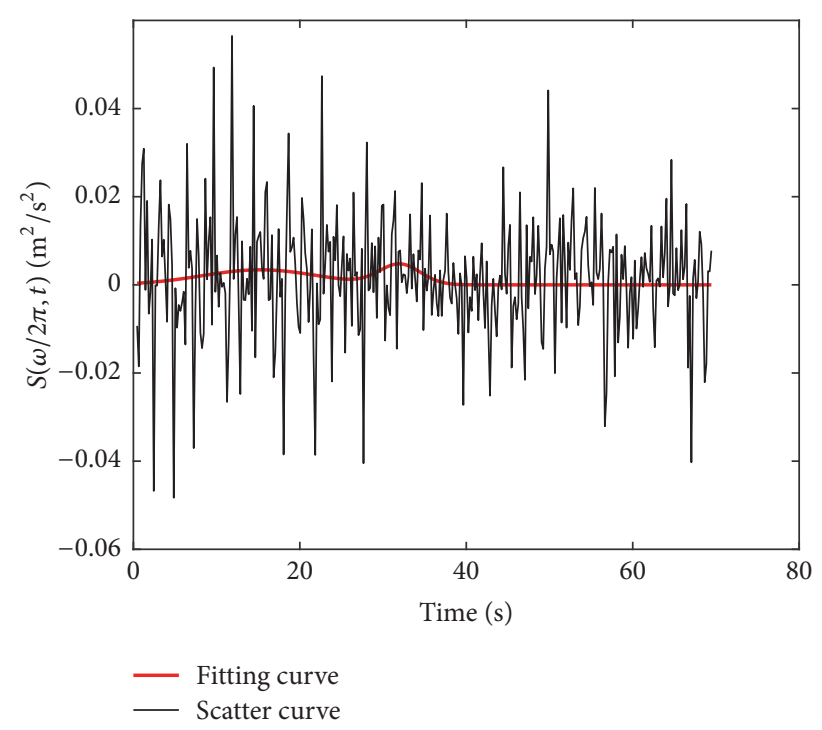

(b) Chi-Chi

FIGURE 7: Fitting of parameter $S_{0}^{(2)}(t)$.

TABLE 1: Values of parameters $\omega_{g}^{(1)}(t), \omega_{g}^{(2)}(t)$.

\begin{tabular}{llcccccc}
\hline Seismic wave & $\omega_{g}^{(k)}(t)$ & $F_{1}$ & $a_{1}$ & $b_{1}$ & $F_{2}$ & $a_{2}$ \\
\hline \multirow{2}{*}{ 8244 Orion } & $\omega_{g}^{(1)}(t)$ & 0.1221 & 20.47 & 11.04 & 0.0244 & 68.71 & 58.09 \\
& $\omega_{g}^{(2)}(t)$ & 1.3729 & 7.203 & 7.891 & 0.1214 & 41.25 \\
Chi-Chi & $\omega_{g}^{(1)}(t)$ & 0.01536 & 20.89 & 5.934 & 0.01417 & 32.22 \\
& $\omega_{g}^{(2)}(t)$ & 0.02761 & 20.74 & 2.6 & 0.03893 & 33.45 \\
\hline
\end{tabular}

TABLE 2: Fitted time-varying expressions for $S_{0}^{(2)}(t)$.

\begin{tabular}{lc}
\hline Seismic wave & $S_{0}^{(2)}(t)$ \\
\hline 8244 Orion & $0.8768 * \exp \left(-(t-20.08 / 4.13)^{2}\right)+0.4321 * \exp \left(-(t-3.329 / 7.39)^{2}\right)$ \\
Chi-Chi & $0.00341 * \exp \left(-(t-15.23 / 9.9)^{2}\right)+0.004576 * \exp \left(-(t-32.02 / 3.495)^{2}\right)$ \\
\hline
\end{tabular}

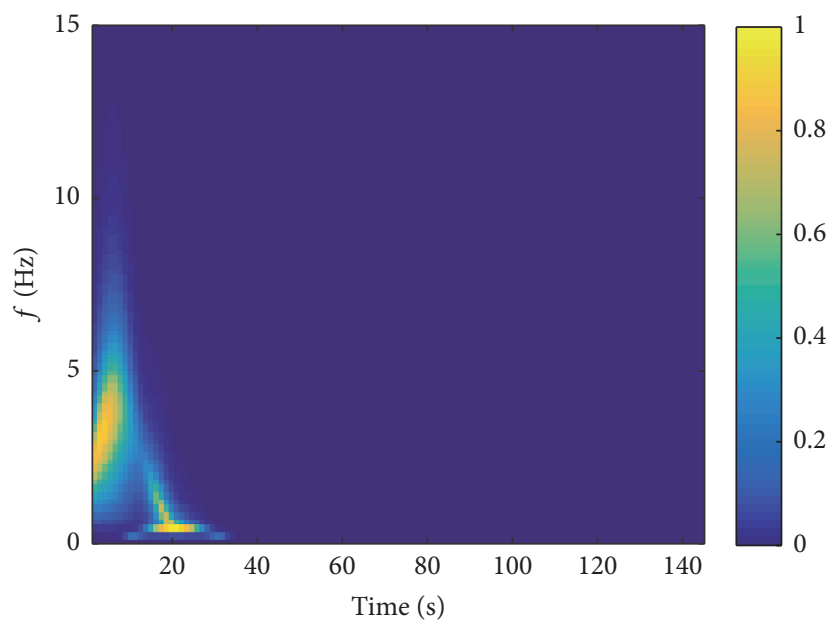

(a) 8244 Orion

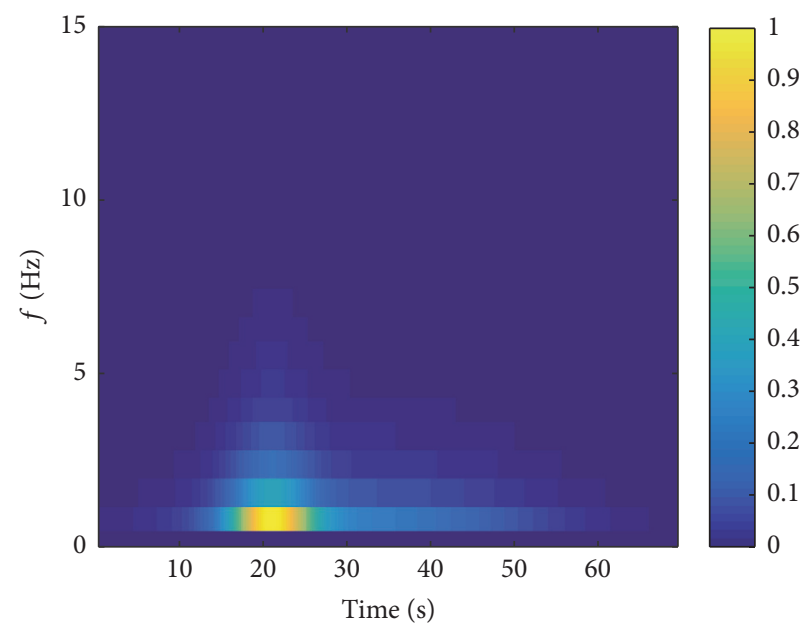

(b) Chi-Chi

FIGURE 8: Time-varying power spectra of the ground motions from the bimodal Kanai-Tajimi model inverse calculation. 


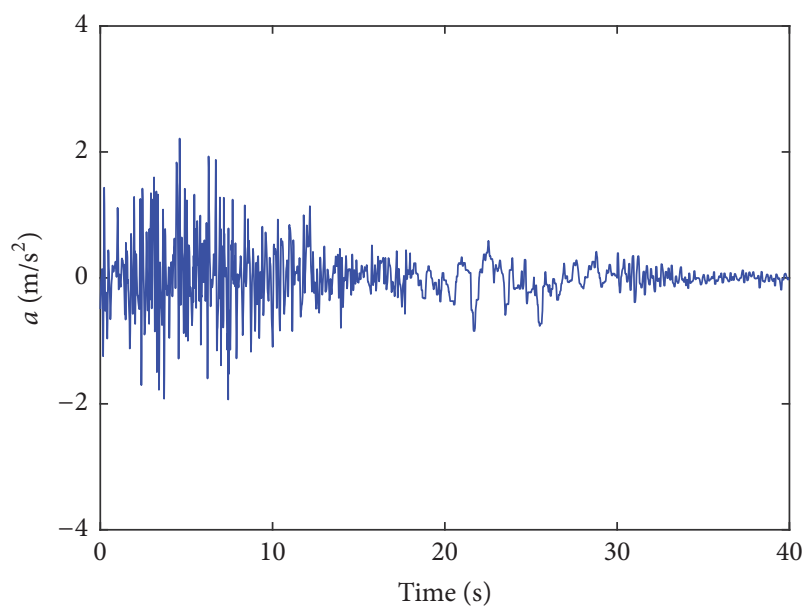

(a) 8244 Orion

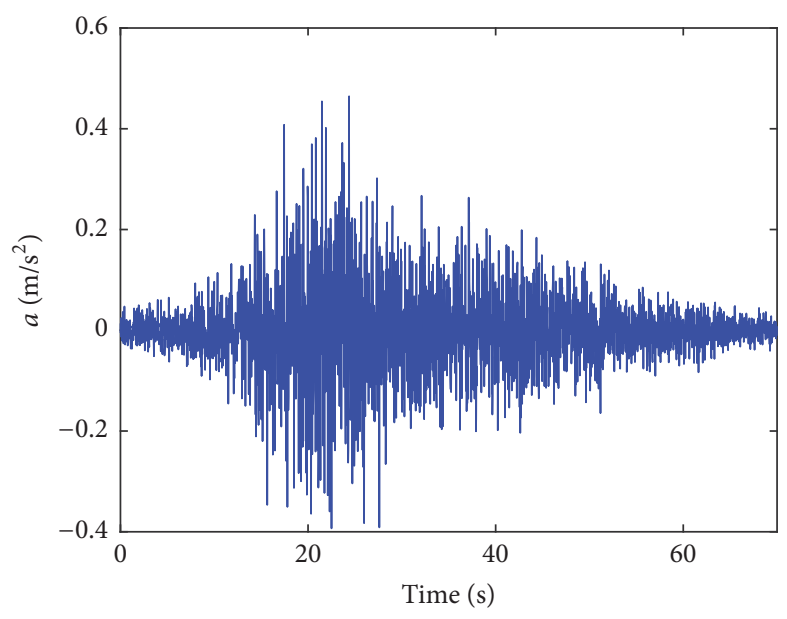

(b) Chi-Chi

FIgURE 9: Acceleration time-history of the simulated ground motion.

and its parameters is analyzed using the time-varying power spectrum-based stochastic process simulation method proposed by Z.-J. Liu and L. Liu [13].

4.1. Time-Varying Power Spectrum-Based Stochastic Process Simulation Method for Ground Motion. Z.-J. Liu and L. Liu [13] introduced a random function-based expression for standard orthogonal random variable and created a sample function set that could generate a stochastic process via a discrete set of representative points of a basic random variable. When this method is applied to simulating nonstationary time-frequency ground motion, the expression is

$$
\begin{aligned}
& \ddot{x}(t) \\
& \quad=\sum_{i=1}^{N} \sqrt{2 S\left(\omega_{i}, t_{i}\right) \triangle \omega}\left[\cos \left(\omega_{i} t\right) X_{i}+\sin \left(\omega_{i} t\right) Y_{i}\right],
\end{aligned}
$$

where $\ddot{x}(t)$ is the time-history of the simulated ground motion, $S\left(\omega_{i}, t_{i}\right)$ is the ground motion's time-varying power spectrum, and $X_{i}$ and $Y_{i}$ are standard orthogonal random variables that satisfy the basic condition shown in

$$
\begin{aligned}
E\left[X_{i}\right] & =E\left[Y_{i}\right]=0, \\
E\left[X_{i} Y_{i}\right] & =0, \\
E\left[X_{i} X_{j}\right] & =E\left[Y_{i} Y_{j}\right]=\delta_{i j},
\end{aligned}
$$

where $E[\cdot]=0$ represents the mathematical expectation and $\delta_{i j}$ is the Kronecker delta.

In an actual stochastic process simulation, the random variables $X_{k}$ and $Y_{k}(k=1,2, \ldots, N)$ are defined as functions of a basic random variable, $\Theta$; that is, the following random functions are constructed:

$$
\begin{aligned}
& X_{k}=\sqrt{2} \cos \left(k \Theta+\frac{\pi}{4}\right), \\
& Y_{k}=\sqrt{2} \sin \left(k \Theta+\frac{\pi}{4}\right),
\end{aligned}
$$

$$
k=1,2, \ldots, N \text {, }
$$

where the basic random variable $\Theta$ is evenly distributed on $[-\pi, \pi]$. It is easy to verify that the standard orthogonal random variables $X_{k}$ and $Y_{k}(k=1,2, \ldots, N)$ constructed in (20) satisfy the basic condition required by (18).

4.2. Simulation Verification of Ground Motion. Based on the parameters identified for the bimodal time-varying modified Kanai-Tajimi models of the 8244 Orion and Chi-Chi earthquakes obtained in Section 2.3, time-varying power spectra, $S\left(\omega_{i}, t_{i}\right)$, for the two earthquakes are calculated using (11). On this basis as well as the method of representing nonstationary stochastic process spectra defined in (18)-(20), the simulated time-histories of the two earthquakes shown in Figures 9(a) and 9 (b) are obtained. Figure 10 shows comparisons of the strength envelope functions and the normalized cumulative energies, respectively, of the simulated and original ground motions. These figures show that the simulated and original ground motions are generally consistent in terms of their nonstationary characteristics in the time domain. To compare the nonstationary characteristics of the simulated and original ground motions in the frequency domain, Figure 11 shows a comparison of the cumulative zero-crossing rates for the simulated and original ground motions. These graphs show that the cumulative zero-crossing rates of the simulated and original ground motions are generally consistent, which means that the improved bimodal time-varying modified Kanai-Tajimi model accurately reflects the nonstationary frequency characteristics of the original ground motion. 


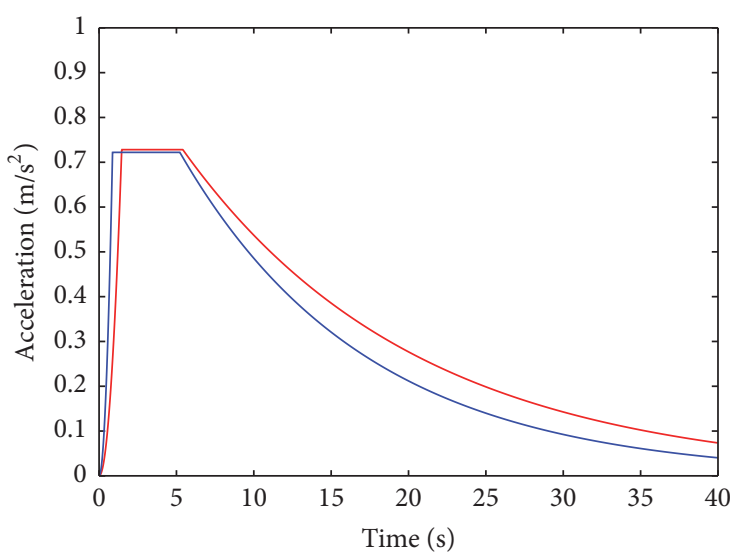

_ Actual earthquake ground motion

_ Simulated earthquake ground motion

(a) 8244 Orion

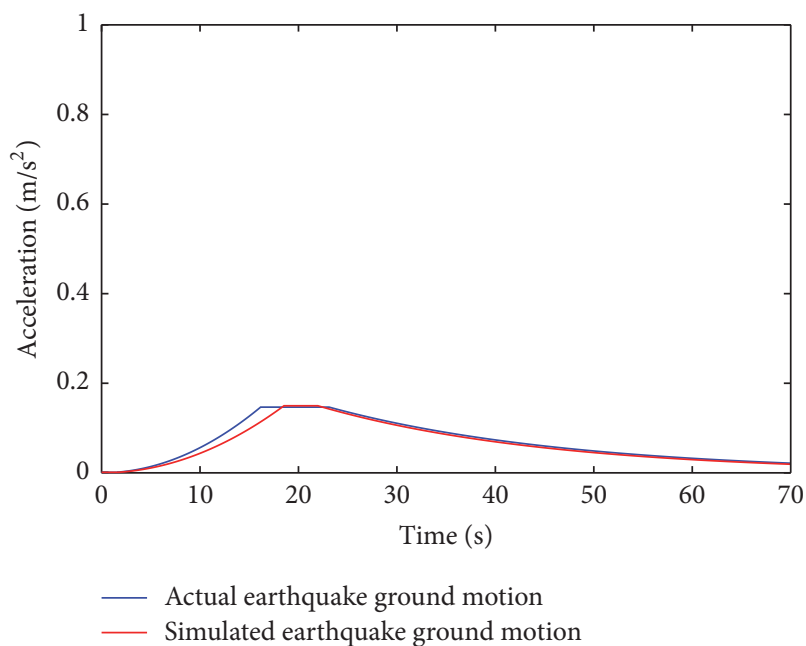

(b) Chi-Chi

FIGURE 10: Comparison of the strength envelopes.

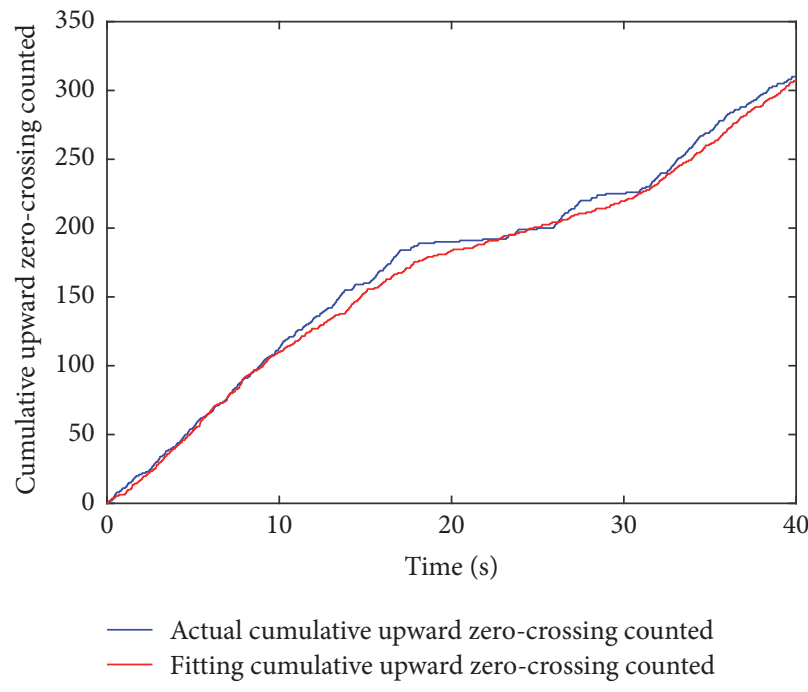

(a) 8244 Orion

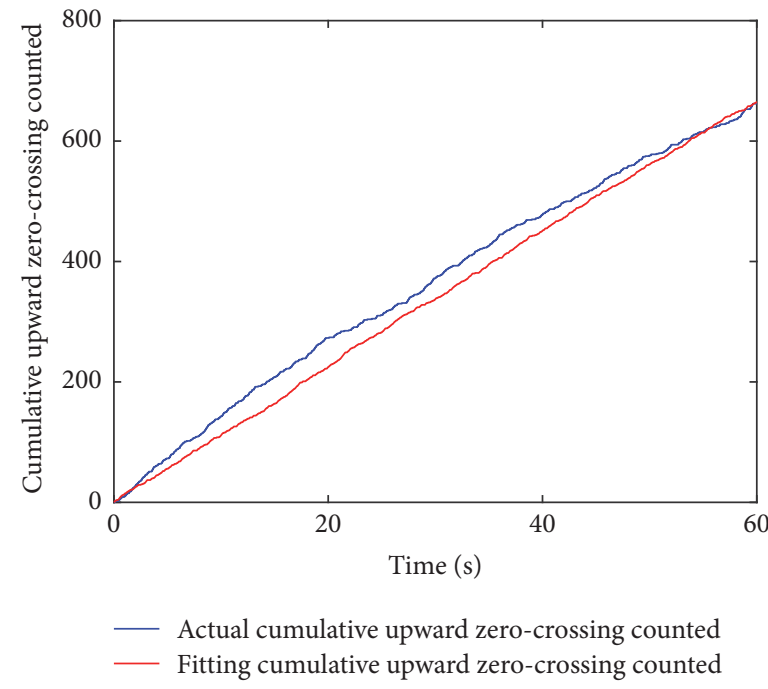

(b) Chi-Chi

FIGURE 11: Comparison of the cumulative upward zero-crossing rates.

\section{Conclusions}

In this paper, the Kanai-Tajimi power spectrum filtering method proposed by Du Xiuli et al. is employed to improve the bimodal time-varying modified Kanai-Tajimi power spectral model proposed by Vlachos et al. A genetic algorithm is employed to identify the optimal model parameters and to establish a time-varying power spectrum parameterization model of the ground motion. As examples of the parameter identification process, time-varying power spectral models of the 8244 Orion and Chi-Chi ground motions and simulation verification of these ground motions show that the improved bimodal time-varying modified Kanai-Tajimi power spectral model accurately describes the nonstationary time-frequency characteristics of ground motion. But the proposed bimodal model is unable to reproduce accelerogram with the strong nonstationarity in frequency content and must be improved in the future. These research findings provide important references for the design of ground motion inputs for seismic analyses of major engineering structures.

\section{Conflicts of Interest}

The authors declare that they have no conflicts of interest.

\section{Acknowledgments}

This work was supported by the National Natural Science Foundation of China under Grant no. 51478068. 


\section{References}

[1] C.-H. Yeh and Y. K. Wen, "Modeling of nonstationary ground motion and analysis of inelastic structural response," Structural Safety, vol. 8, no. 1-4, pp. 281-298, 1990.

[2] H. Cao, H. Yang, M. I. Friswell, and S. Bai, "The Analysis of Earthquake Waves Based on Nonlinear Responses of RC Structures," in Proceedings of the ASME 7th Biennial Conference on Engineering Systems Design and Analysis, pp. 69-74, Manchester, England, 2004.

[3] J. P. Conte, "Effects of earthquake frequency nonstationary on inelastic structural response," in Proceedings of the 10th WCEE, vol. 7, pp. 3645-3651, Madrid, Spain, 1992.

[4] J. Wang, L. Fan, S. Qian, and J. Zhou, "Simulations of nonstationary frequency content and its importance to seismic assessment of structures," Earthquake Engineering and Structural Dynamics, vol. 31, no. 4, pp. 993-1005, 2002.

[5] M. B. Priestley, "Power spectral analysis of non-stationary random processes," Journal of Sound and Vibration, vol. 6, no. 1, pp. 86-97, 1967.

[6] H. Kameda, "Evolutionary spectra of seismogram by multifilter," Journal of Engineering Mechanism Division. American Society of Civil Emgineering, vol. 101, no. EM6, pp. 787-801, 1975.

[7] M. Sugito, F. Oka, and A. Yashima, "Strong motion prediction on rock surface by superposed evolutionary spectra," in Proceedings of the 12th World Conference on Earthquake Engineering, vol. 2111, pp. 1-8, 2000.

[8] G. Deodatis, "Non-stationary stochastic vector processes: seismic ground motion applications," Probabilistic Engineering Mechanics, vol. 11, no. 3, pp. 149-168, 1996.

[9] J. Fan and Z. Zeng, "Establishment of time variable modified Kanai-Tajimi non-stationary stochastic model of Strong ground motion record," Acta Seismologica Sinica, vol. 32, no. 6, pp. 733743,2010

[10] Z. Liu, L. Wang, Q. Dan, Y. Zhou, and B. Tian, "Generalized evolutionary spectrum of non-stationary ground motion and its applications in seismic design of hydraulic structures," Journal of Hydraulic Engineering, vol. 46, no. 9, pp. 1028-1036, 2015.

[11] C. Vlachos, K. G. Papakonstantinou, and G. Deodatis, "A multi-modal analytical non-stationary spectral model for characterization and stochastic simulation of earthquake ground motions," Soil Dynamics and Earthquake Engineering, vol. 80, pp. 177-191, 2016.

[12] D. Xiu-Li and C. Hou-Qun, "Random simulation and its parameter determination method of earthquake ground motion," Earthquake Engineering and Engineering Vibration, vol. 14, no. 4, pp. 1-5, 1994.

[13] Z.-J. Liu and L. Liu, "Simulation of stochastic ocean states by random function methods," Journal of Vibration and Shock, vol. 33, no. 20, pp. 1-6, 2014.

[14] M. Shinozuka, "Random process with evolutionary power. J. of the Engineering Mechanics Division," ASCE, vol. 96, no. EM4, pp. 543-555, 1970.

[15] M. Shinozuka and G. Deodatis, "Stochastic process models for earthquake ground motion," Probabilistic Engineering Mechanics, vol. 3, no. 3, pp. 114-123, 1988.

[16] K. Kanai, Semi-empirical formula for the seismic characteristics of the ground, Bulletin of the Earthquake Research Institute, University of Tokyo, Tokyo, Japan, 1957.

[17] H. Tajimi, "A statistical model of determining the maximum response of a steucture during an earthquake," in Proceedings of the 2nd WCEE, Tokyo, Japan, 1960.
[18] J. Jin-Ren, L. Qin-Nian, and S. Jing-Jiang, "Statistical properties of strong motion spectrum," Research report 87-032, Institute of Engineering Mechanics, China Earthquake Administration, 1987.

[19] Z. Min-Zheng, Estimation of near-fault earthquake engineering parameters [Doctoral dissertation], Institute of Engineering Mechanics, China Earthquake Administration, 1986.

[20] A. Mijatovic, Solving Optimal Power Flow with Voltage Constraints Using MATLAB Optimization Toolbox, Murdoch University, 2013.

[21] W. Ying, Synthetic of seismic acceleration graphs [MS thesis], Institute of Engineering Mechanics, China Earthquake Administration, 1987.

[22] J. H. Holland, Adaptation in Natural and Artificial Systems: An Introductory Analysis with Applications to Biology, Control, and Artificial Intelligence, University of Michigan Press, Oxford, UK, 1975. 


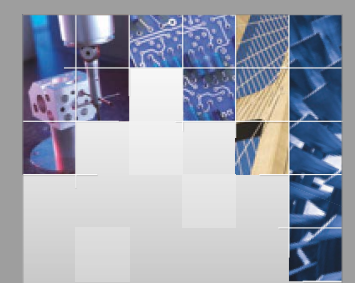

\section{Enfincering}
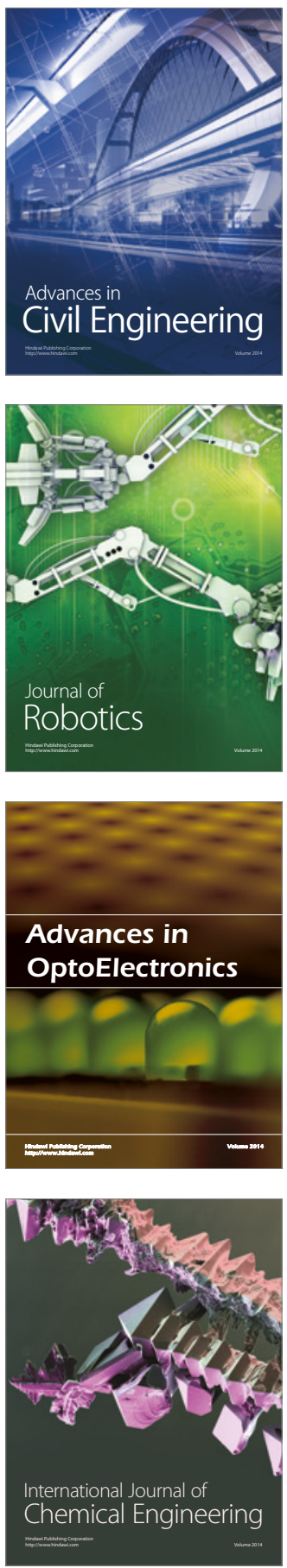

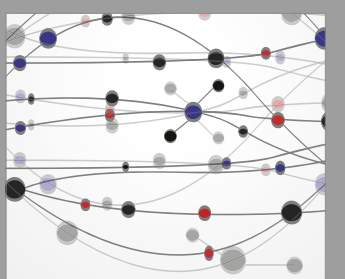

The Scientific World Journal

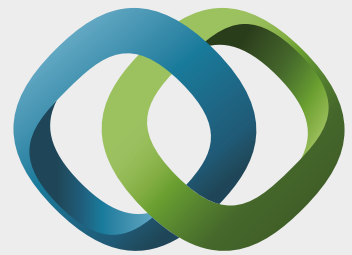

\section{Hindawi}

Submit your manuscripts at

https://www.hindawi.com
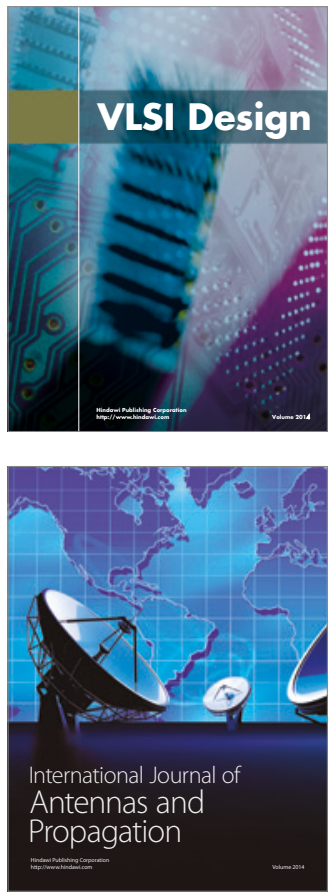

\section{Rotating}

Machinery
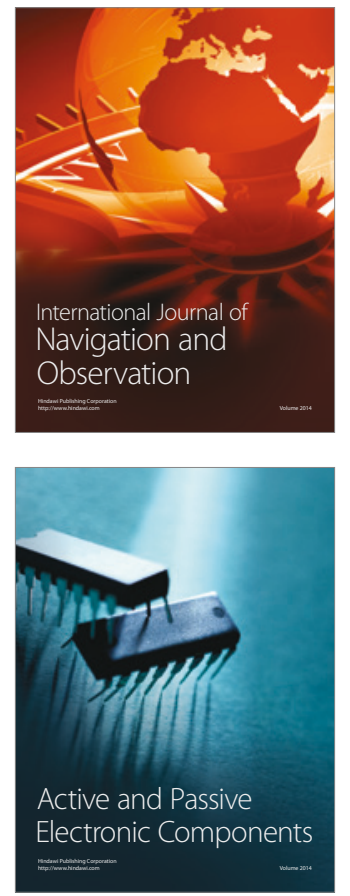
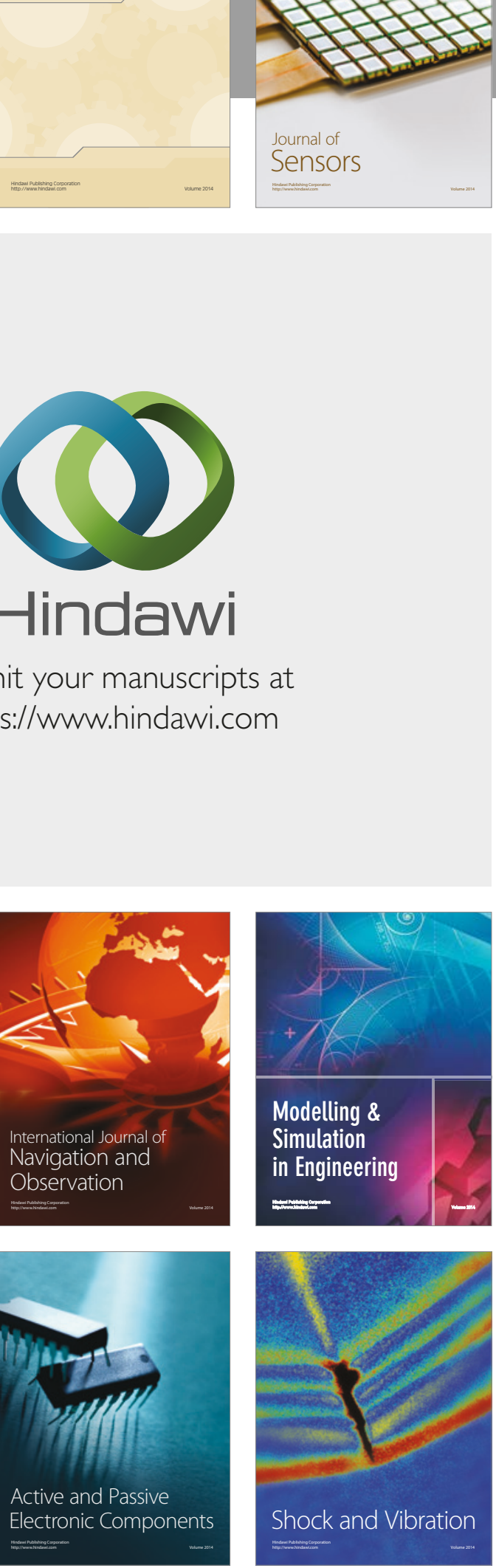
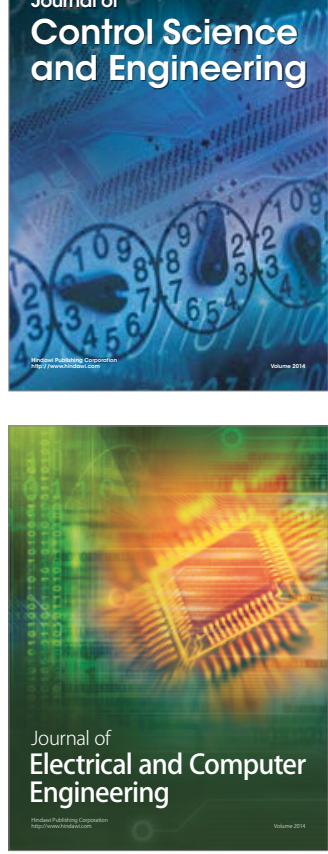

Distributed

Journal of

Control Science

and Engineering
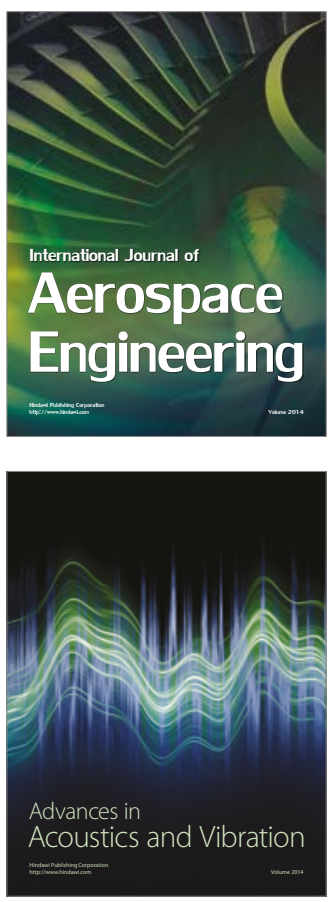

Sensor Networks 